\title{
THE RATIO OF INVARIANT METRICS ON THE ANNULUS AND THETA FUNCTIONS
}

\author{
KAZUO AZUKAWA \\ Department of Mathematics, Toyama University \\ Gofuku, Toyama 930, Japan
}

Introduction. Let $A=A_{q}$ be the annulus with parameter $q \in(0,1)$ :

$$
A_{q}=\{\lambda \in \mathbb{C} ; q<|\lambda|<1\} .
$$

Let $C^{A}, K^{A}$, and $P^{A}$ be the Carathéodory, the Kobayashi, and the P-metric on $A$, respectively (for the definition of $P^{A}$ see Section 1). Since all the metrics $C^{A}, K^{A}$, and $P^{A}$ are invariant for biholomorphic mappings and since $A$ is one-dimensional, the functions $C P^{A}(\lambda):=C^{A}(X) / P^{A}(X)$ and $K P^{A}(\lambda):=K^{A}(X) / P^{A}(X)$ for $X$ a non-zero holomorphic tangent vector at $\lambda \in A$ are well-defined as functions on $A$ and invariant for holomorphic automorphisms of $A$.

The main purpose of this paper is to show the following.

Theorem A. Let $r \in(0,1)$ be defined by

$$
\frac{\log q}{\pi i}=\frac{\pi i}{-\log r} .
$$

For every $\lambda \in A=A_{q}$ with $v \in(0,1)$ such that

$$
|\lambda|=q^{v}
$$

we have

$$
\begin{aligned}
& C P^{A}(\lambda)=\prod_{n \geq 1} \frac{\left|e^{2 \pi i v}+r^{2 n-1}\right|^{2}}{\left(1+r^{2 n-1}\right)^{2}}, \\
& K P^{A}(\lambda)=\prod_{n \geq 1} \frac{\left|e^{2 \pi i v}-r^{2 n}\right|^{2}}{\left(1-r^{2 n}\right)^{2}} .
\end{aligned}
$$

1991 Mathematics Subject Classification: Primary 32H15; Secondary 32F05.

Partially supported by the Grant-in-Aid for Scientific Research, the Ministry of Education, Science and Culture, Japan.

The paper is in final form and no version of it will be published elsewhere. 
Consequently, the functions $\alpha:(0,1) \ni v \mapsto C P^{A}\left(q^{v}\right) \in[0,+\infty)$ and $\beta:(0,1) \ni$ $v \mapsto K P^{A}\left(q^{v}\right) \in[0,+\infty)$ are unimodal; moreover, $\alpha$ (resp. $\beta$ ) is strictly decreasing (resp. increasing) in $(0,1 / 2)$ and strictly increasing (resp. decreasing) in $(1 / 2,1)$; therefore,

$$
\begin{aligned}
& \min C P^{A}=\min \alpha=\alpha(1 / 2)=\prod_{n \geq 1} \frac{\left(1-r^{2 n-1}\right)^{2}}{\left(1+r^{2 n-1}\right)^{2}}, \\
& \max K P^{A}=\max \beta=\beta(1 / 2)=\prod_{n \geq 1} \frac{\left(1+r^{2 n}\right)^{2}}{\left(1-r^{2 n}\right)^{2}} .
\end{aligned}
$$

Assertion (0.4) appeared in the proof of Proposition 3.4 in [2] and its proof in this paper is different from that in [2], which comes from Myrberg's theorem on the Green function of a hyperbolic Riemann surface. The argument of this paper is based on the theory of theta functions attached to the tori $\mathbb{T}(1, \tau)=\mathbb{C} /(\mathbb{Z}+\tau \mathbb{Z})$ and $\mathbb{T}(1,-1 / \tau)=\mathbb{C} /(\mathbb{Z}+(-1 / \tau) \mathbb{Z})$, where $\tau \in H=\{\tau \in \mathbb{C} ; \operatorname{Im} \tau>0\}$ is the number given by

$$
\tau=\frac{\log q}{\pi i}, \quad-\frac{1}{\tau}=\frac{\log r}{\pi i}
$$

(see $(0.1))$. In fact, the functions $C P^{A}$ and $K P^{A}$ are directly represented by a ratio of theta functions attached to the torus $\mathbb{T}(1,-1 / \tau)$ (Theorem $\mathrm{C}$ in Section 3 ).

Theorem A is important because as its consequence we get the following wellknown fact: All holomorphic automorphisms of $A$ consist of the functions $(\lambda \mapsto$ $\left.e^{i \theta} \lambda\right)_{\theta \in \mathbb{R}}$ and $\left(\lambda \mapsto e^{i \theta} q / \lambda\right)_{\theta \in \mathbb{R}}$. Indeed, let $C_{s}=\{\lambda \in A ;|\lambda|=s\}$ for $s \in(q, 1)$. Since the functions $r: A \ni \lambda \mapsto q / \lambda \in A$ and $A \ni \lambda \mapsto e^{i \theta} \lambda \in A$ for $\theta \in \mathbb{R}$ are automorphisms of $A$, we see that $C P^{A}$ is constant on each $C_{s}$ and that $C P^{A}\left(C_{s}\right)=C P^{A}\left(C_{q / s}\right)$. Let $\varphi$ be a holomorphic automorphism of $A$. Theorem A implies that for every $s \in(q, 1), \varphi\left(C_{s}\right)$ coincides with $C_{s}$ or $C_{q / s}$. Since the function $(q, 1) \ni s \mapsto|\varphi(s)| \in(q, 1)$ is a homeomorphism, it follows that either $\varphi\left(C_{s}\right)=C_{s}$ for all $s$, or $\varphi\left(C_{s}\right)=C_{q / s}$ for all $s$. Assume first that $\varphi\left(C_{s}\right)=C_{s}$ for all $s$. Then the function $\varphi(\lambda) / \lambda$ has modulus 1 on $A$ so that $\varphi(\lambda)=e^{i \theta} \lambda, \lambda \in A$ for some real $\theta$. If $\varphi\left(C_{s}\right)=C_{q / s}$ for all $s$, then the last argument implies that $r \circ \varphi(\lambda)=e^{i \theta} \lambda, \lambda \in A$ for some real $\theta$, as desired.

We also obtain the representation of $C P^{A}$ in terms of the Green function of $A$.

Theorem B. If $G^{A}(\cdot, \lambda)$ is the Green function on $A$ with pole at $\lambda \in A$, then

$$
C P^{A}(\lambda)=\exp \left(-G^{A}(-q / \bar{\lambda}, \lambda)\right)
$$

for $\lambda \in A$.

The author would like to thank Professor S. Egami for his helpful suggestion on the subject of this note. This work was partially done in the discussion in the Complex Analysis Semester, Warsaw in October, 1992. The author is very grateful to the staff of the Banach Center for their heartfelt hospitality. 
1. Invariant metrics on the annulus. For a point $p \in M$ of a complex manifold $M$, we define a subspace $P S^{M}(p)$ of the space $N P S(M)$ of all negative plurisubharmonic functions on $M$ as follows:

$$
P S^{M}(p)=\{f \in N P S(M) ; f(q)-\log \|z(q)-z(p)\| \leq O(1) \text { as } q \rightarrow p\},
$$

where $z$ is a holomorphic coordinate around $p$ and \|\| means the complex euclidian norm on $\mathbb{C}^{m}, m=\operatorname{dim} M$. Here, we assume the function $-\infty$ identically belongs to $N P S(M)$. The definition of $P S^{M}(p)$ does not depend on the choice of the coordinate $z$. For $q \in M$, let

$$
u_{p}^{M}(q)=u^{M}(q, p)=\sup \left\{f(q) ; f \in P S^{M}(p)\right\} .
$$

The function $u_{p}^{M}$ is called the pluri-complex Green function with pole at $p$ (cf. [14], [9], [1], [2], [3], [6], [10], [8], [11]).

Let $X \in T_{p} M$ be a holomorphic tangent vector at $p \in M$. Let $E=\{\lambda \in$ $\mathbb{C} ;|\lambda|<1\}$ be the unit disk in $\mathbb{C}$. Taking a holomorphic function $\varphi$ from an $\varepsilon$-neighborhood $\varepsilon E$ of 0 in $\mathbb{C}$ to $M$ with $\varphi(0)=p$ and $\varphi^{\prime}(0)=X$, we define

$$
P^{M}(X)=\limsup _{\lambda \rightarrow 0, \lambda \neq 0} \frac{\exp \circ u_{p}^{M} \circ \varphi(\lambda)}{|\lambda|}
$$

(cf. [1], [2], [6], [10], [11]). The definition of $P^{M}(X)$ does not depend on the choice of $\varphi$ (cf. [2], [6]), and the function $P^{M}$ is a pseudo-metric on $M$, that is, $P^{M}$ is $[0,+\infty)$-valued on the holomorphic tangent bundle $T M$ satisfying $P^{M}(\lambda X)=$ $|\lambda| P^{M}(X)$ for any $X \in T M$ and $\lambda \in \mathbb{C}$. The assignment $M \mapsto P^{M}$ of pseudometrics possesses the decreasing property, i.e., for a holomorphic mapping $\Phi$ from $M$ to $M^{\prime}, P^{M^{\prime}}\left(\Phi_{*} X\right) \leq P^{M}(X)$ for all $X \in T M$ and the metric $P^{E}$ for the unit disk $E$ in $\mathbb{C}$ coincides with the Poincaré metric on $E$, which implies that if $C^{M}$ and $K^{M}$ denote the Carathéodory and the Kobayashi pseudo-metrics respectively, then $C^{M} \leq P^{M} \leq K^{M}$ for any complex manifold $M$ (cf. [1], [2], [6]). Furthermore, if by $I S^{M}(p)=\left\{X \in T_{p} M ; S^{M}(X)<1\right\}$ we denote the indicatrix at $p \in M$ for a pseudo-metric $S^{M}$ on $M$, then the following are well-known:

(1) $I C^{M}(p)$ is convex for all $p \in M([5])$.

(2) $I P^{M}(p)$ is pseudoconvex for all $p \in M([2])$.

(3) $I K^{M}(p)$ is not necessarily pseudoconvex ([7]).

If $M$ is a hyperbolic Riemann surface, then the function $-u_{p}^{M}$ is the usual Green function $G^{M}(\cdot, p)$ of $M$ with pole at $p$ (cf. [9], [1]). Let $z$ be a holomorphic coordinate around $p$ and $\mu(d / d z)_{p}, \mu \in \mathbb{C}$, be a holomorphic tangent vector at $p$. If

$$
\varphi:=z^{-1} \circ(\varepsilon E \ni \lambda \mapsto z(p)+\mu \lambda \in \mathbb{C}): \varepsilon E \rightarrow M,
$$

then $\varphi(0)=p$ and $\varphi^{\prime}(0)=\varphi_{*}\left((d / d \lambda)_{0}\right)=\mu(d / d z)_{p}$, so that

$$
P^{M}\left(\mu\left(\frac{d}{d z}\right)_{p}\right)=|\mu|\left|\frac{d \exp \circ u_{p}^{M} \circ z^{-1}(z(p)+\lambda)}{d \lambda}\right|_{\lambda=0} \mid .
$$


It is well-known ([17], [9], [2]) that the pluri-complex Green function $u^{A}$ on the annulus $A=A_{q}$ is given by

$$
u_{\lambda}^{A}(\mu)=(1-v) \log |\mu|+\log \left|\Theta_{\lambda}(\mu)\right| \quad(\lambda, \mu \in A)
$$

where

$$
\Theta_{\lambda}(\mu)=\frac{\prod_{n \geq 1}\left(1-q^{2 n} \mu / \lambda\right)\left(1-q^{2 n-2} \lambda / \mu\right)}{\prod_{n \geq 1}\left(1-q^{2 n-2} \bar{\lambda} \mu\right)\left(1-q^{2 n} /(\bar{\lambda} \mu)\right)}
$$

and $v=v(\lambda) \in(0,1)$ with

$$
q^{v}=|\lambda|
$$

It follows from (1.1) that

$$
P^{A}\left(\left(\frac{d}{d \lambda}\right)_{\lambda}\right)=\frac{q^{-v^{2}} \prod_{n \geq 1}\left(1-q^{2 n}\right)^{2}}{\prod_{n \geq 1}\left(1-q^{2 n-2+2 v}\right)\left(1-q^{2 n-2 v}\right)} .
$$

We note that the Kobayashi metric $K^{A}$ on $A$ coincides with the usual Poincaré metric on $A$ by virtue of the following fact ([6], [13]): If $\pi: N \rightarrow M$ is a (not necessarily universal) covering of a complex manifold $M$, then $K^{M}\left(\pi_{*} X\right)=K^{N}(X)$ for all $X \in T M$. Let $H=\{\eta \in \mathbb{C} ; \operatorname{Im} \eta>0\}$ be the upper half plane in $\mathbb{C}$. Since the mapping $H \ni \eta \mapsto e^{\tau \log \eta} \in A$ with

$$
\tau=\frac{\log q}{\pi i}
$$

is a covering on $A([2])$, and since $|d \eta| /(2 \operatorname{Im} \eta)$ is the Poincaré metric on $H$, we see

$$
K^{A}\left(\left(\frac{d}{d \lambda}\right)_{\lambda}\right)=\frac{\pi}{(-2 \log q) q^{v} \sin \pi v}
$$

for $\lambda \in A$ with $v$ as in (1.3).

Concerning the Carathéodory metric $C^{A}$ on $A$, the following is well-known ([17], [2]): For $\lambda \in A$ with $v$ in (1.3),

$$
C^{A}\left(\left(\frac{d}{d \lambda}\right)_{\lambda}\right)=\frac{\prod_{n \geq 1}\left(1-q^{2 n}\right)^{2}\left(1+q^{2 n-1+2 v}\right)\left(1+q^{2 n-1-2 v}\right)}{\prod_{n \geq 1}\left(1+q^{2 n-1}\right)^{2}\left(1-q^{2 n-2+2 v}\right)\left(1-q^{2 n-2 v}\right)} .
$$

2. Theta functions and their transformation formulas. By $\mathbb{T}\left(\omega_{1}, \omega_{2}\right)$ we denote the torus $\mathbb{C} /\left(\omega_{1} \mathbb{Z}+\omega_{2} \mathbb{Z}\right)$ with basic periods $\left(\omega_{1}, \omega_{2}\right)$ satisfying $\omega_{2} / \omega_{1} \in H$; the number $\omega_{2} / \omega_{1}$ is called the modulus of the torus $\mathbb{T}\left(\omega_{1}, \omega_{2}\right)$. For $\tau \in H$ and $v \in \mathbb{C}$, let

$$
\begin{aligned}
& \theta_{0}^{*}(v, \tau)=2 e^{\frac{\pi i \tau}{4}} \prod_{n \geq 1}\left(1-e^{2 n \pi i \tau}\right)\left(1-e^{2 \pi i(n \tau+v)}\right)\left(1-e^{2 \pi i(n \tau-v)}\right) \\
& \theta_{0}(v, \tau)=(\sin \pi v) \theta_{0}^{*}(v, \tau) \\
& \theta_{3}(v, \tau)=\prod_{n \geq 1}\left(1-e^{2 n \pi i \tau}\right)\left(1+e^{2 \pi i((n-1 / 2) \tau+v)}\right)\left(1+e^{2 \pi i((n-1 / 2) \tau-v)}\right)
\end{aligned}
$$


(cf. [4, p. 69]). Then, the functions $\theta_{j}(\cdot, \tau)(j=0,3)$ are two of four theta functions attached to the torus $\mathbb{T}(1, \tau)$ and satisfy

$$
\begin{array}{ll}
\theta_{0}(v+1, \tau)=-\theta_{0}(v, \tau), & \theta_{0}(v+\tau, \tau)=-q^{-1} e^{-2 \pi i v} \theta_{0}(v, \tau), \\
\theta_{3}(v+1, \tau)=\theta_{3}(v, \tau), & \theta_{3}(v+\tau, \tau)=q^{-1} e^{-2 \pi i v} \theta_{3}(v, \tau)
\end{array}
$$

(cf. [4, pp. 58, 64]).

Since we have holomorphic isomorphisms

$$
\mathbb{T}\left(1, \omega_{2} / \omega_{1}\right) \cong \mathbb{T}\left(\omega_{1}, \omega_{2}\right) \cong \mathbb{T}\left(-\omega_{2}, \omega_{1}\right) \cong \mathbb{T}\left(1,-\omega_{1} / \omega_{2}\right),
$$

the second one of which comes from the mapping $\mathbb{C} \ni \lambda \mapsto \lambda-\omega_{1} \in \mathbb{C}$, if $\tau \in$ $H$, then $\mathbb{T}(1, \tau) \cong \mathbb{T}(1,-1 / \tau)$. We need the transformation formulas connecting $\theta_{j}(\cdot, \tau)$ and $\theta_{j}(\cdot,-1 / \tau)$ for $j=0,3$. If $v \in \mathbb{C}$ and $\tau \in H$, then

$$
\begin{aligned}
& \theta_{0}(v,-1 / \tau)=-i e^{\pi i \tau v^{2}} \sqrt{\tau / i} \theta_{0}(\tau v, \tau), \\
& \theta_{3}(v,-1 / \tau)=e^{\pi i \tau v^{2}} \sqrt{\tau / i} \theta_{3}(\tau v, \tau),
\end{aligned}
$$

where the square root is taken so that $\sqrt{\tau / i}=1$ for $\tau=i$ (cf. [4, pp. 73, 75]).

3. Proof of Theorem A and Theorem B. We first show the following.

Theorem C. Let $\tau \in H$ be defined by

$$
q=e^{\pi i \tau} .
$$

For $\lambda \in A$ with $v=v(\lambda) \in(0,1)$ such that

$$
|\lambda|=q^{v}
$$

we have

$$
\begin{aligned}
C P^{A}(\lambda) & =\frac{\theta_{3}(v,-1 / \tau)}{\theta_{3}(0,-1 / \tau)}, \\
K P^{A}(\lambda) & =\frac{\theta_{0}^{*}(v,-1 / \tau)}{\theta_{0}^{*}(0,-1 / \tau)} .
\end{aligned}
$$

We note that (3.1) is equivalent to $(0.5)$.

Proof of Theorem C. Items (1.4) and (1.6) imply that

$$
C P^{A}(\lambda)=\frac{q^{v^{2}} \prod_{n \geq 1}\left(1+q^{2 n-1-2 v}\right)\left(1+q^{2 n-1+2 v}\right)}{\prod_{n \geq 1}\left(1+q^{2 n-1}\right)^{2}}
$$

Using (2.1) to get

$$
\theta_{3}(\tau v, \tau)=\prod_{n \geq 1}\left(1-q^{2 n}\right)\left(1+q^{2 n-1+2 v}\right)\left(1+q^{2 n-1-2 v}\right),
$$

we have

$$
C P^{A}(\lambda)=\frac{q^{v^{2}} \theta_{3}(\tau v, \tau)}{\theta_{3}(0, \tau)}
$$


By the transformation formula (2.4) we have

$$
\theta_{3}(v,-1 / \tau)=q^{v^{2}} \sqrt{\tau / i} \theta_{3}(\tau v, \tau)
$$

therefore, assertion (3.3) follows.

Similarly, items (1.4) and (1.5) imply that

$$
K P^{A}(\lambda)=\frac{\pi}{-\log q} \frac{q^{v^{2}}}{2 q^{v} \sin \pi v} \frac{\prod_{n \geq 1}\left(1-q^{2 n-2+2 v}\right)\left(1-q^{2 n-2 v}\right)}{\prod_{n \geq 1}\left(1-q^{2 n}\right)^{2}} .
$$

Since $\sin \pi \tau v=\left(1-q^{2 v}\right) /\left(2 i q^{v}\right)$, it follows that

$$
K P^{A}(\lambda)=\frac{q^{v^{2}}}{\tau} \frac{\sin \pi \tau v}{\sin \pi v} \frac{\prod_{n \geq 1}\left(1-q^{2 n+2 v}\right)\left(1-q^{2 n-2 v}\right)}{\prod_{n \geq 1}\left(1-q^{2 n}\right)^{2}} .
$$

Using (2.1) to get

$$
\theta_{0}^{*}(\tau v, \tau)=2 q^{1 / 4} \prod_{n \geq 1}\left(1-q^{2 n}\right)\left(1-q^{2 n+2 v}\right)\left(1-q^{2 n-2 v}\right),
$$

we have

$$
K P^{A}(\lambda)=\frac{q^{v^{2}}}{\tau} \frac{\sin \pi \tau v}{\sin \pi v} \frac{\theta_{0}^{*}(\tau v, \tau)}{\theta_{0}^{*}(0, \tau)} .
$$

By the transformation formula (2.3) we have

$$
\theta_{0}^{*}(v,-1 / \tau) \sin \pi v=-i q^{v^{2}} \sqrt{\tau / i}(\sin \pi \tau v) \theta_{0}^{*}(\tau v, \tau) .
$$

Dividing both sides by $\sin \pi v$ and taking the limit as $v \rightarrow 0$, we see

$$
\theta_{0}^{*}(0,-1 / \tau)=-i \sqrt{\tau / i} \tau \theta_{0}^{*}(0, \tau)
$$

so that we get

$$
\frac{\theta_{0}^{*}(v,-1 / \tau)}{\theta_{0}^{*}(0,-1 / \tau)}=\frac{\sin \pi \tau v}{\sin \pi v} \frac{q^{v^{2}}}{\tau} \frac{\theta_{0}^{*}(\tau v, \tau)}{\theta_{0}^{*}(0, \tau)} .
$$

Combining this with (3.7) we obtain formula (3.4) and complete the proof of Theorem C.

We shall show Theorem A stated in Introduction.

Proof of Theorem A. By virtue of (3.2), using the definition (2.2) of $\theta_{3}(\cdot,-1 / \tau)$, noticing the fact

$$
r=e^{-2 \pi i / \tau}
$$

(see $(0.1))$, we have

$$
C P^{A}(\lambda)=\frac{\prod_{n \geq 1}\left(1+r^{2 n-1} e^{2 \pi i v}\right)\left(1+r^{2 n-1} e^{-2 \pi i v}\right)}{\prod_{n \geq 1}\left(1+r^{2 n-1}\right)^{2}} .
$$

Since $\overline{e^{2 \pi i v}}=e^{-2 \pi i v}$ because $v$ is real, we have obtained assertion (0.3) in Theorem A. 
Similarly, by virtue of (3.3), using (2.1) we have

$$
K P^{A}(\lambda)=\frac{\prod_{n \geq 1}\left(1-r^{2 n} e^{2 \pi i v}\right)\left(1-r^{2 n} e^{-2 \pi i v}\right)}{\prod_{n \geq 1}\left(1-r^{2 n}\right)^{2}},
$$

and assertion (0.4) in Theorem A. The proof is complete.

Proof of Theorem B. We first note that the Green function $G^{A}(\cdot, \lambda)$ of $A$ with pole at $\lambda \in A$ coincides with $-u_{\lambda}^{A}$ (see Section 1). It follows from (1.2) that

$$
\begin{aligned}
\exp \left(-G^{A}(-q / \bar{\lambda}, \lambda)\right) & =\exp u^{A}(-q / \bar{\lambda}, \lambda) \\
& =\frac{q^{(1-v)^{2}} \prod_{n \geq 1}\left(1+q^{2 n} q /|\lambda|^{2}\right)\left(1+q^{2 n-2}|\lambda|^{2} / q\right)}{\prod_{n \geq 1}\left(1+q^{2 n-1}\right)^{2}} \\
& =\frac{q^{v^{2}} \prod_{n \geq 1}\left(1+q^{2 n-1-2 v}\right)\left(1+q^{2 n-1+2 v}\right)}{\prod_{n \geq 1}\left(1+q^{2 n-1}\right)^{2}}
\end{aligned}
$$

By virtue of (3.5) we have proved the desired assertion of Theorem B.

\section{References}

[1] K. Azukawa, Two intrinsic pseudo-metrics with pseudoconvex indicatrices and starlike circular domains, J. Math. Soc. Japan 38 (1986), 627-647.

[2] —, The invariant pseudo-metric related to negative plurisubharmonic functions, Kodai Math. J. 10 (1987), 83-92.

[3] E. Bedford and B. A. Taylor, Plurisubharmonic functions with logarithmic singularities, Ann. Inst. Fourier (Grenoble) 38 (1988), 133-171.

[4] K. Chandrasekharan, Elliptic Functions, Springer, New York, 1985.

[5] C. Carathéodory, Über die Geometrie der analytischen Abbildungen, die durch analytische Funktionen von zwei Veränderlichen vermittelt werden, Abh. Math. Sem. Univ. Hamburg 6 (1928), 96-145.

[6] S. Dineen, The Schwarz Lemma, Clarendon Press, Oxford, 1989.

[7] J. E. Fornaess and B. Stensøness, Lectures on Counterexamples in Several Complex Variables, Princeton Univ. Press, Princeton, 1987.

[8] M. Jarnicki and P. Pflug, Invariant pseudodistances and pseudometrics-Completeness and product property, Ann. Polon. Math. 55 (1991), 169-189.

[9] M. Klimek, Extremal plurisubharmonic functions and invariant pseudodistances, Bull. Soc. Math. France 113 (1985), 231-240.

[10] - Infinitesimal pseudo-metrics and the Schwarz lemma, Proc. Amer. Math. Soc. 105 (1989), 134-140.

[11] —, Pluripotential Theory, Clarendon Press, Oxford, 1991.

[12] S. Kobayashi, Hyperbolic Manifolds and Holomorphic Mappings, Marcel Dekker, New York, 1970

[13] - Intrinsic distances, measures and geometric function theory, Bull. Amer. Math. Soc. 82 (1976), 357-416.

[14] L. Lempert, La métrique de Kobayashi et la représentation des domaines sur la boule, Bull. Soc. Math. France 109 (1981), 427-474. 
[15] E. A. Poletskiŭ and B. V. Shabat, Invariant metrics, in: Encyclopedia of Mathematical Sciences 9, G. M. Khenkin (ed.), 1989, 63-111.

[16] H. L. Royden, Remarks on the Kobayashi metric, in: Lecture Notes in Math. 185, Springer, Berlin, 1971, 125-137.

[17] R. R. Simha, The Carathéodory metric of the annulus, Proc. Amer. Math. Soc. 50 (1975), 162-166. 